\title{
Resectional surgery combined with chemotherapy remains the treatment of choice for multidrug-resistant tuberculosis
}

\author{
Yuji Shiraishi, MD \\ Yutsuki Nakajima, MD \\ Naoya Katsuragi, MD \\ Makoto Kurai, MD \\ Nobumasa Takahashi, MD
}

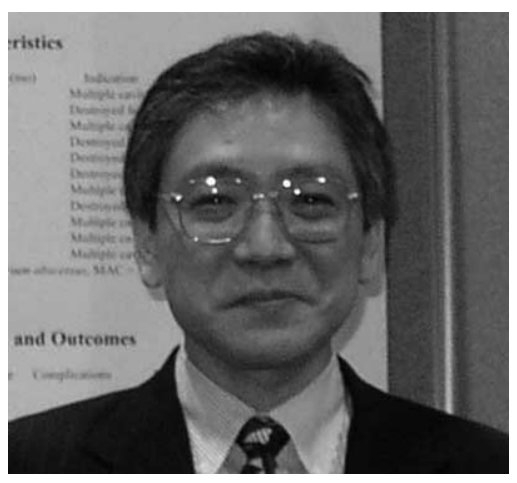

Dr Shiraishi
From the Section of Chest Surgery, Fukujuji Hospital, Tokyo, Japan.

Read at the Eighty-fourth Annual Meeting of The American Association for Thoracic Surgery, Toronto, Ontario, Canada, April 25-28, 2004.

Received for publication April 11, 2004; revisions received May 23, 2004; accepted for publication June 16, 2004.

Address for reprints: Yuji Shiraishi, MD, Section of Chest Surgery, Fukujuji Hospital, 3-1-24 Matsuyama, Kiyose, Tokyo, 204-8522 Japan (E-mail: yujishi@mvb. biglobe.ne.jp)

J Thorac Cardiovasc Surg 2004;128:523-8

$0022-5223 / \$ 30.00$

Copyright (C) 2004 by The American Association for Thoracic Surgery

doi:10.1016/j.jtcvs.2004.06.012
Objective: Multidrug-resistant tuberculosis remains a significant health problem. The best available treatment for multidrug-resistant tuberculosis is the combination of pulmonary resection and antituberculous chemotherapy. We herein report the results of pulmonary resection combined with chemotherapy for multidrug-resistant tuberculosis at our institution during the years 2000 through 2002.

Methods: Between 1983 and 2002, 87 patients underwent 95 pulmonary resections for multidrug-resistant tuberculosis. Of these, the 30 (34\%) patients operated on from January 1, 2000, to December 31, 2002, are reviewed in the present study. All patients were maintained on multidrug regimens preoperatively and postoperatively. Indications for surgical intervention included persistently positive sputum and a high risk of relapse. Thirty-three pulmonary resections were performed, consisting of pneumonectomy $(\mathrm{n}=12)$, lobectomy $(\mathrm{n}=17)$, and segmentectomy $(\mathrm{n}=4)$. The bronchial stump was reinforced with a latissimus dorsi muscle flap in 29 resections.

Results: There was no operative mortality. Bronchopleural fistulas occurred in 2 patients. Five patients had a space problem. All patients attained sputum-negative status after the operation. Relapse occurred in 3 patients: 2 had a relapse at the bronchial stump, and the remaining patient had a relapse in the postlobectomy space. One late death occurred. Of the 29 survivors, 27 (93\%) were free from disease, with a median follow-up of 24 months (range, 8-47 months).

Conclusions: An increasing number of patients with multidrug-resistant tuberculosis are requiring resectional surgery in the 21 st century. Pulmonary resection combined with chemotherapy achieves high cure rates with acceptable morbidity and remains the treatment of choice for multidrug-resistant tuberculosis.

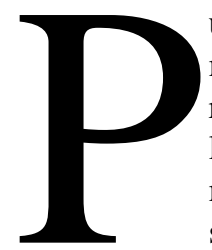
ulmonary tuberculosis remains a global threat. The emergence of multidrug-resistant strains (ie, strains resistant to at least isoniazid and rifampin [INN: rifampicin] $)^{1}$ is an especially significant health problem. ${ }^{2,3}$ Multidrug-resistant tuberculosis is difficult to treat, and its management thus requires expertise. Medical treatment with first- and second-line antituberculous drugs does not achieve satisfactory outcomes. ${ }^{4}$ Therefore resection of cavitary lesions or destroyed lobes, which harbor large numbers of bacilli, has been advocated to improve the efficacy of medical treatment. ${ }^{5-10}$ Although the results of medical treatment of multidrug-resistant tuberculosis have improved since the introduction of fluoroquinolone antibiotics, ${ }^{11,12}$ current fluoroquinolone-containing regimens are not panaceas. No randomized controlled studies have been conducted to compare the results of chemotherapy alone with the results of pulmonary resection combined with chemotherapy. ${ }^{13}$ Retrospective studies during the fluoroquinolone era, however, have demonstrated 
TABLE 1. Types of pulmonary resection

\begin{tabular}{lrrr}
\hline Procedure & Right & Left & Total \\
\hline Completion pneumonectomy & 1 & 0 & 1 \\
Pneumonectomy & 5 & 6 & 11 \\
Upper and middle lobectomy & 1 & 0 & 1 \\
Upper lobectomy with segmentectomy* & 4 & 1 & 5 \\
Upper lobectomy & 5 & 5 & 10 \\
Lower lobectomy & 0 & 1 & 1 \\
Segmentectomy & 1 & 3 & 4 \\
Total & 17 & 16 & 33 \\
\hline
\end{tabular}

*Upper lobectomy with resection of superior segment of the lower lobe.

that surgical treatment plus chemotherapy achieves more favorable results than chemotherapy alone. ${ }^{12,14}$ The combination of pulmonary resection and antituberculous chemotherapy, when feasible, thus represents the best available treatment of multidrug-resistant tuberculosis.

Our institution was established as a sanatorium in the late 1940s and is recognized as a major center for pulmonary tuberculosis in Japan. We have performed pulmonary resections since 1983 on patients with multidrug-resistant tuberculosis. As a result of a recent increase in the number of referrals, patients operated on after January 1, 2000, comprise one third of our entire surgical series. Furthermore, during this period, we have been using newer fluoroquinolones in multidrug treatment regimens and performing muscle flap reinforcement of the bronchial stump in almost all of our pulmonary resections. We herein report the results of pulmonary resection combined with chemotherapy for multidrug-resistant tuberculosis at our institution during the years 2000 through 2002.

\section{Patients and Methods}

Between January 1983 and December 2002, 87 patients underwent 95 pulmonary resections for multidrug-resistant tuberculosis at Fukujuji Hospital. Of these 87 patients, the 30 (34\%) patients who were operated on from January 1, 2000, to December 31, 2002, are reviewed in the present study. There were 21 (70\%) male patients and $9(30 \%)$ female patients. Age at the time of the operation ranged from 22 to 64 years (median, 48 years). None of the patients were immunocompromised. Twelve patients had diabetes. Sputum smears and sputum cultures were performed on admission. Drug susceptibility testing was routinely performed on positive cultures in our laboratory. Bronchoscopy was done in some cases to rule out contralateral disease, coexisting malignancies, or both.

All patients had received antituberculous chemotherapy at the referral hospitals or at our institution, and at the time of admission, were shedding strains resistant to a median of 6 drugs (range, 2-9 drugs), including isoniazid and rifampin. Multidrug regimens, with a median of 4 drugs (range, 3-6 drugs), were initiated in all patients. Drugs included in the regimens were chosen on the basis of drug susceptibility testing results. When the regimens being used in the referral hospitals were appropriate, those regimens were continued. Fluoroquinolones were included in the regimens of $24(80 \%)$ patients.
Eighteen patients, 5 patients, and 1 patient received levofloxacin, sparfloxacin, and ciprofloxacin, respectively. Other drugs used included ethambutol, streptomycin, kanamycin, enviomycin, pyrazinamide, ethionamide, para-aminosalicylic acid, cycloserine, and thioacetazone. Patients were treated with multidrug regimens for at least 3 months. Resection of cavitary lesions or destroyed lobes was considered for those patients who had persistently positive sputum despite chemotherapy. Among patients who had sputum conversion from positive to negative in response to chemotherapy, surgical intervention was considered for those at high risk of relapse on the basis of drug resistance patterns, radiographic findings, or both.

Preoperative studies included chest roentgenography, computed tomographic scans of the chest, pulmonary function tests, arterial blood gas analysis, and a quantitative perfusion scan. To be eligible for surgical intervention, patients had to have sufficient pulmonary reserve to tolerate pulmonary resection and have lesions predominantly localized to one lung. Scattered nodular lesions on the contralateral side were acceptable; indeed, only 6 patients had their tuberculosis limited entirely to one side, with the opposite side totally intact. When patients had cavitary lesions on both sides, staged bilateral resections were applied if patients could tolerate bilateral procedures. Thirty-three pulmonary resections were performed (Table 1). Three patients underwent staged multiple resections. One was a patient who had undergone a right upper lobectomy with segmentectomy and who subsequently underwent a right completion pneumonectomy for recurrent disease. The remaining 2 patients had staged bilateral resections: a left upper lobectomy and a right upper lobectomy in 1 patient and a right upper lobectomy and resection of 2 segments of the left lung in the other patient.

Operations were performed after achievement of general anesthesia and epidural anesthesia with the use of a double-lumen endobronchial tube. After induction of anesthesia, we performed a posterolateral thoracotomy. In patients with dense adhesions, we chose extrapleural dissection to keep their cavities intact. During the course of extrapleural dissection, we used electrocautery, bipolar scissors (PowerStar Bipolar Scissors; Ethicon Inc, Somerville, NJ), or both to reduce blood loss. ${ }^{15}$ The bronchus was divided and closed with staples $(n=29)$ or sutures $(n=4)$. The bronchial stump was reinforced with a latissimus dorsi muscle flap in 29 resections and with a pericardial fat pad in one resection. Bronchial stump reinforcement was not performed in 3 segmental resections. Our technique for constructing a latissimus dorsi muscle flap was the same as the technique described by Pairolero and associates. ${ }^{16}$ We meticulously used electrocautery and an argon beam coagulator to stop all internal bleeding. The pleural cavity was irrigated with at least $10 \mathrm{~L}$ of saline and povidone iodine, and the chest was then drained of fluid.

After the operation, all patients were maintained on multidrug regimens, generally the same as their preoperative regimens. Duration of postoperative chemotherapy ranged from 6 to 30 months (median, 18 months). Patients were followed up through December 31, 2003, with a median follow-up of 24 months (range, 8-47 months). Operative mortality included all deaths clearly related to the operation, regardless of the postoperative interval. All cases of bronchopleural fistula or empyema occurring subsequent to the date of the operation were considered postoperative complications. 


\section{Results}

Ten (33\%) patients had positive sputum at the time of the operation. Surgical operations were equally divided between the right side and the left side (Table 1). Operating time ranged from 140 to 623 minutes (median, 270 minutes). Median intraoperative blood loss was $180 \mathrm{~mL}$ (range, 10-1330 mL). No spillage of the contents of cavities into the operative field occurred in any patient. There was no operative mortality. Nine (30\%) patients had a total of 10 complications (Table 2).

Two patients had bronchopleural fistulas; both had diabetes and positive sputum at the time of the operation. The first patient had strains resistant to 7 drugs and underwent a right pneumonectomy with latissimus dorsi muscle flap. His sputum culture, which was negative after the operation, turned positive 3 months after the operation. However, no lesions were identified in the left lung, and the bronchial stump seemed intact. Two months later, bronchial stump disruption occurred, and lavage fluid from the bronchial stump was positive for tuberculosis. His strains had acquired resistance to 2 additional drugs used in the regimen by this time. He underwent an open-window thoracostomy. Over time, the muscle flap covering the bronchial stump was gradually eroded by the uncontrollable tuberculous infection, and a bronchopleural fistula finally developed 12 months after the initial operation, resulting in tuberculous empyema. The second patient had strains resistant to 8 drugs, and underwent a left upper lobectomy with stapling technique. He spit up the staples 21 months after the operation, and his sputum turned positive at that time. His strains had acquired resistance to 2 additional drugs used in the regimen by this time. Bronchopleural fistula and empyema occurred 2 months afterward. He then underwent an openwindow thoracostomy.

Among the 5 patients in whom a space problem occurred, 3 patients had undergone an upper lobectomy with segmentectomy, and 1 patient had undergone an upper and middle lobectomy. The operative records of these 4 patients contained comments that the surgeons had great difficulty dissecting dense pleural adhesions and that the remaining lobes were relatively small and stiff. The fifth patient had undergone resections of both the anterior segment of the left upper lobe and the superior segment of the left lower lobe. The space problems in all 5 patients were treated with thoracoplasty.

All 30 study patients attained negative sputum status after the operation. Relapse of multidrug-resistant tuberculosis occurred in 3 patients. Two patients had a relapse at the bronchial stump 3 and 21 months postoperatively, respectively, as mentioned above. The third patient had a relapse in the postlobectomy space. This patient underwent a right upper lobectomy with segmentectomy, followed by a thoracoplasty for a space problem 1 month later. After thora-
TABLE 2. Postoperative complications

\begin{tabular}{lc}
\hline Postoperative complications & $\begin{array}{c}\text { No. of } \\
\text { patients (\%) }\end{array}$ \\
\hline Bronchopleural fistula with empyema & $2(6)$ \\
Space problem & $5(15)$ \\
Prolonged air leak & $2(6)$ \\
Chylothorax & $1(3)$ \\
\hline
\end{tabular}

coplasty, 4 months after the initial operation, this patient had a space problem again. A computed tomographic scan of the chest showed a small cavity in the right lower lobe that had perforated into the space. The pleural fluid collected from the space was positive for tuberculosis. The patient was treated with completion pneumonectomy after 6 months of open drainage, and negative conversion was achieved. One late death resulted from cerebral infarction 8 months postoperatively. Twenty-seven (93\%) of the 29 survivors were free from disease at the time of follow-up.

\section{Discussion}

The recent emergence of tubercle bacilli resistant to both isoniazid and rifampin, which are the most potent antituberculous drugs, represents an emerging global problem. ${ }^{2,3}$ These multidrug-resistant strains of tuberculosis are of great concern. ${ }^{1}$ Medical treatment of multidrug-resistant tuberculosis is not satisfactory, even with long-term aggressive and intensive multidrug regimens, including first- and secondline drugs. ${ }^{4}$ Adjunctive surgical treatment has been advocated to improve the efficacy of medical treatment. ${ }^{5-10}$ The purpose of such surgical treatment is to reduce the bacterial burden by resecting cavitary lesions or destroyed lobes. The introduction of fluoroquinolone antibiotics has raised hopes of improving the currently dismal prognosis for multidrugresistant tuberculosis. However, antituberculous chemotherapy containing fluoroquinolones are not panaceas. Resectional surgery continues to have an important adjunctive role in the management of multidrug-resistant tuberculosis.

In the year 2000, our hospital was certified as one of the key centers for treating multidrug-resistant tuberculosis in Japan. This certification has brought about an increasing number of referrals for treatment of patients with multidrugresistant tuberculosis and has altered the temporal distribution of patients in our multidrug-resistant tuberculosis surgical series. Of the 87 patients treated surgically over the 20 -year period from 1983 through 2002, one third (30 patients) were operated on from 2000 through 2002. The present study focused on these 30 patients, who received our most current surgical and medical treatment regimen for multidrug-resistant tuberculosis. During this period, we have been using more potent fluoroquinolones in our multidrug regimens to improve the efficacy of medical treat- 
ment. We also used muscle flaps in almost all resections to prevent bronchial stump problems and space problems.

Our results were basically in line with those of previous studies. ${ }^{7-10}$ Patients were shedding strains resistant to a median of 6 drugs, including isoniazid and rifampin, and they had been treated with multidrug regimens containing a median of 4 drugs for at least 3 months before the operation. Indications for resectional operations were (1) persistent positive sputum despite chemotherapy or (2) high risk of relapse, even after negative conversion was achieved by means of chemotherapy. Dissection of dense pleural adhesions was performed extrapleurally. ${ }^{17}$ Operative mortality was zero, but morbidity was a problem. Postoperative chemotherapy was continued for a median of 18 months. Although the follow-up period is relatively short, pulmonary resection has achieved high cure rates.

The distinctive features of the present study included active use of levofloxacin in the multidrug regimens and a higher incidence of bronchopleural fistulas and space problems than reported in previous studies. This study included only patients operated on after January 1, 2000. As a result, levofloxacin, which was used in $60 \%$ of the patients, was the most commonly used fluoroquinolone in the multidrug regimens. In the study by Chan and associates, ${ }^{14}$ which reviewed 205 patients treated between 1984 and 1998, ofloxacin was used in 124 patients, and levofloxacin was used in only 9 patients. In a study from Turkey, ofloxacin was the fluoroquinolone included in the regimens. ${ }^{12}$ Levofloxacin has been reported to be more efficacious than ofloxacin in the treatment of multidrugresistant tuberculosis. ${ }^{18}$ In the present study one third of the patients had positive sputum at the time of the operation. This figure is lower than what has been previously reported. In the study by Pomerantz and associates, ${ }^{10}$ one half of the patients had positive sputum at the time of the operation. Our higher preoperative negative conversion rate might be in part caused by more patients having been treated with levofloxacincontaining regimens.

Despite the use of a latissimus dorsi muscle flap, 2 (6\%) patients had bronchopleural fistulas in the present study. This fistula rate was higher than the rate reported in previous studies. In the study by Pomerantz and associates, ${ }^{10}$ only 5 bronchopleural fistulas occurred after 180 pulmonary resections. In a report from South Africa, 2 bronchial stump fistulas occurred in 35 patients undergoing pneumonectomy, and no fistulas occurred after lobectomies. ${ }^{8}$ We believe that the development of fistulas in our study was not caused by problems with our surgical technique. The fistulas occurred late after the operation and were preceded by a relapse at the bronchial stump. Both patients initially achieved negative conversion after the operation, but their strains at the time of relapse had acquired resistance to 2 additional drugs used in the regimens. We suspect that the bronchial stump might have been contaminated with tuber- cle bacilli at the time of the operation and that the infection could not be controlled with postoperative intensive chemotherapy, resulting in bronchial stump disruption. Even the latissimus dorsi muscle flap could not protect the stump against uncontrollable tuberculous infection. We fully agree with the following criterion for pulmonary resection proposed by Iseman and coworkers ${ }^{5}$ : "sufficient drug activity to diminish the mycobacterial burden enough to facilitate probable healing of the bronchial stump."

We also encountered space problems, even with the use of a latissimus dorsi muscle flap. Four of the 5 space problems occurred after procedures that were more extensive than lobectomy, such as bilobectomy or lobectomy with segmentectomy. Furthermore, in these 4 patients we encountered great difficulty dissecting dense pleural adhesions. As a result, the remaining lobes were too small and too stiff to fill the pleural space. In these cases putting the latissimus dorsi muscle flap into the pleural space might not have been sufficient to obliterate the entire residual space. A better technique for harvesting the flap and covering both the bronchial stump and the raw surface of the remaining lung needs to be developed. Tailored thoracoplasty might be indicated to achieve further obliteration of the residual space. Although pneumonectomy produces a cleaner operation, we cannot conclude that pneumonectomy would have been the procedure of choice for these patients. Unless the lung is totally destroyed, it is better to leave as much lung parenchyma as possible without jeopardizing the completeness of the removal of major lesions to preserve pulmonary functions.

This retrospective study has its limitations. It was not possible to conduct a randomized controlled study to compare the efficacy of surgical treatment plus chemotherapy with the efficacy of chemotherapy alone. Therefore our study was a single-arm study. The patients enrolled in this study had already been selected as eligible for pulmonary resection. We have achieved a $93 \%$ cure rate in our surgical group to date, but this figure cannot be directly compared with cure rates for study groups receiving chemotherapy alone. Our current strategy for the management of multidrug-resistant tuberculosis is to perform pulmonary resection whenever indicated to augment the efficacy of medical treatment. A group in Denver has taken the same strategy and has achieved favorable results. ${ }^{5,14}$ In their recent report the use of surgical intervention increased in frequency during the course of their study period, and surgical resection was performed on $83 \%$ of the patients treated during the last 5 years. ${ }^{14}$ Our results and previously reported results indicate that patients with multidrug-resistant tuberculosis could have a greater chance of being cured with the use of adjuvant resectional surgery in combination with intensive medical treatment. 


\section{References}

1. Iseman MD. Treatment of multidrug-resistant tuberculosis. $N$ Engl J Med. 1993;329:784-91.

2. World Health Organization. Anti-tuberculosis drug resistance in the world. Report no. 3. The WHO/IUATLD global project on antituberculosis drug resistance surveillance 1999-2002. Geneva: World Health Organization; 2004.

3. Nachega JB, Chaisson RE. Tuberculosis drug resistance. A global threat. Clin Infect Dis. 2003;36(suppl 1):S24-30.

4. Goble M, Iseman MD, Madsen LA, Waite D, Ackerson L, Horsburgh CR Jr. Treatment of 171 patients with pulmonary tuberculosis resistant to isoniazid and rifampin. $N$ Engl J Med. 1993;328:527-32.

5. Iseman MD, Madsen L, Goble M, Pomerantz M. Surgical intervention in the treatment of pulmonary disease caused by drug-resistant Mycobacterium tuberculosis. Am Rev Respir Dis. 1990;141:623-5.

6. Pomerantz M, Madsen L, Goble M, Iseman M. Surgical management of resistant mycobacterial tuberculosis and other mycobacterial pulmonary infections. Ann Thorac Surg. 1991;52:1108-12.

7. Treasure RL, Seaworth BJ. Current role of surgery in Mycobacterium tuberculosis. Ann Thorac Surg. 1995;59:1405-9.

8. Van Leuven M, De Groot M, Shean KP, Von Oppell UO, Willcox PA. Pulmonary resection as an adjunct in the treatment of multiple drugresistant tuberculosis. Ann Thorac Surg. 1997;63:1368-73.

9. Sung S-W, Kang CH, Kim YT, Han SK, Shim Y-S, Kim JH. Surgery increased the chance of cure in multi-drug resistant pulmonary tuberculosis. Eur J Cardiothorac Surg. 1999;16:187-93.

10. Pomerantz BJ, Cleveland JC Jr, Olson HK, Pomerantz M. Pulmonary resection for multi-drug resistant tuberculosis. J Thorac Cardiovasc Surg. 2001;121:448-53.

11. Park SK, Kim CT, Song SD. Outcome of chemotherapy in 107 patients with pulmonary tuberculosis resistant to isoniazid and rifampin. Int J Tuberc Lung Dis. 1998;2:877-84.

12. Tahaoglu K, Törün T, Sevim T, Ataç G, Kir A, Karasulu L, et al. The treatment of multidrug-resistant tuberculosis in Turkey. $N$ Engl J Med. 2001;345:170-4.

13. American Thoracic Society/Centers for Disease Control and Prevention/Infectious Diseases Society of America. Treatment of tuberculosis. Am J Respir Crit Care Med. 2003;167:603-62.

14. Chan ED, Laurel V, Strand MJ, Chan JF, Huynh MN, Goble M, et al. Treatment and outcome analysis of 205 patients with multidrug-resistant tuberculosis. Am J Respir Crit Care Med. 2004;169:1103-9.

15. Shiraishi Y, Nakajima Y, Koyama A, Takasuna K, Katsuragi N, Yoshida S. Morbidity and mortality after 94 extrapleural pneumonectomies for empyema. Ann Thorac Surg. 2000;70:1202-6.

16. Pairolero PC, Arnold PG, Piehler JM. Intrathoracic transposition of extrathoracic skeletal muscle. J Thorac Cardiovasc Surg. 1983;86: 809-17.

17. Brown J, Pomerantz M. Extrapleural pneumonectomy for tuberculosis. Chest Surg Clin North Am. 1995;5:289-96.

18. Yew WW, Chan CK, Leung CC, Chau CH, Tam CM, Wong PC, et al. Comparative roles of levofloxacin and ofloxacin in the treatment of multidrug-resistant tuberculosis. Preliminary results of a retrospective study from Hong Kong. Chest. 2003;124:1476-81.

\section{Discussion}

Dr Andrew A. Conlan (Worcester, Mass). Dr Shiraishi, I congratulate you and your team on the very excellent surgical outcome, especially disease control, and on the quality of your presentation. It is a retrospective study, if I take it correctly, and there are 30 patients, 10 of whom were sputum positive coming to surgical intervention and 20 who were judged to be at very high risk of relapse, and therefore surgical intervention was added to their treatment. An excellent disease-free status with an average for a medium follow-up of 28 months is a great achievement.

It is hard to talk about multiple drug-resistant tuberculosis without acknowledging the global problem, and it is staggering that a third of the global population, 2 billion persons, have tuberculosis, with 2 million of these thought have drug-resistant tuberculosis. These costs are staggering. For example, to treat a patient with normal tuberculosis is about $\$ 12,000$ to bring them to a sputum-negative state, and with multiple drug-resistant tuberculosis, it is about $\$ 200,000$ per patient.

I was particularly impressed with the surgical management, and I commend the early and prophylactic use of the extrapleural plane for these very tough lobectomies and pneumonectomies. Likewise, using the latissimus dorsi flap was a very good treatment inclusion.

I would like to ask a number of questions. You had 5 patients with space problems. Interestingly, in your article you mentioned that the surgeons noted tough operating conditions and also that they noted small and inexpansile remaining lung. All of them had resections greater than a lobe. Now this set of circumstances is a classic endgame for a postresection space, and even though a latissimus dorsi flap was put in, I wonder whether you have any comment that if the surgeon recognized this whether he should not have used additional flaps or even considered thoracoplasty at the time of the resection because all of them went into the chronic space, sequential operations, and ultimate thoracoplasty at a much later date. What are your thoughts about that?

Dr Shiraishi. That is a good point. Actually, we encountered 5 patients with space problems. In 4 of the 5 patients, we had great difficulty in dissecting dense pleural adhesions during surgical intervention. Therefore the remaining lung was very small and stiff, and it would be better to obliterate the whole space by means of tailored thoracoplasty or a serratus muscle flap in addition to the latissimus dorsi muscle, but I do not have a good answer for that right now. Another option is that we would take out the whole lung and do a pneumonectomy, but some patients had very poor pulmonary reserve and could not tolerate a pneumonectomy. Therefore we try to leave as much lung parenchyma as possible. We avoid pneumonectomy and perform bilobectomy or lobectomy with segmentectomy.

Dr Conlan. Certainly it is a matter of extreme surgical judgment to pay homage to each of those things you mentioned.

Now your results have been incredibly good, the best. However, I wanted to, as we always do, ask you about the failures. You said 2 of your failures occurred at the bronchial stump. Now I have always had difficulty with this concept of the bronchial stump being the point of failure because unless we have histopathology and biopsy specimens from the bronchial stump that are positive for acid-fast bacilli on stain or on culture, I do not regard it as the primary source. The bronchial stump is broken down, and therefore it is a conduit, and when you wash it and get a positive wash, you are retrieving fluid from the peribronchial space, the pleura, or the parenchyma distal to it. Therefore I wonder whether you want to comment on the bronchial stump being the prime source of recurrence.

Dr Shiraishi. We had 2 relapses at the bronchial stump. One patient had undergone a right pneumonectomy, and his sputum became positive again 4 months after the operation. At that time, his chest computed tomographic scan showed no lesions on the left side, and the bronchial stump looked good. But 2 months later, we checked the stump again and found that the stump was totally disrupted. We could see the flap covering the stump. We washed the stump, and the lavage fluid was positive for tuberculosis. Therefore I conjectured that the relapse occurred at the stump. 
Dr Conlan. I have one last question. I wondered if you could just define for us the residuals that you feel have the highest bacillus or the highest tuberculosis population within them, and do you identify high-risk residuals compared with low-risk residuals? We are often in the position where we remove a destroyed lobe, but we are aware that there are small nodular abnormalities in the contralateral lung or the same lung. What type of lesions do you designate as low risk, and also tell us which ones that you regard as high risk.

Dr Shiraishi. As you know, the cavities or destroyed lobes are at great risk for relapse, but small tiny nodules will not be. Therefore you have to resect cavities or destroyed lobes, but you can leave small nodules.

Dr Stephen D. Cassivi (Rochester, Minn). I appreciated your presentation. Clearly this is a difficult problem, and your results are very commendable.

I have just a very short question. It regards your postresectional antimicrobial therapy and how you choose the length of time that you are going to treat these patients. In your presentation, your range was from 6 months to "upward." How do you choose when to stop antimicrobial therapy?

Dr Shiraishi. Basically, we try to keep patients on multidrug regimens for at least 18 months after sputum conversion has been achieved. Therefore if the patient has experienced negative conversion before the operation, we can shorten the duration of postoperative chemotherapy. Usually, we keep the patients on chemotherapy for at least 18 months after the operation.

Dr Rajan Santosham (Chennai, India). I have one or two comments to make. We do not resect multidrug-resistant tuberculosis unless it is for massive hemoptysis because we are very worried about the outcome, and a large number of our patients become worse off than when they started. They have empyema, bronchopleural fistula, and space problems. But if there is resistant tuberculosis and if it is cavitary, we still perform thoracoplasty, which is very ideal for localized cavitary disease with persistent organisms.

Dr Shiraishi. We sometimes do thoracoplasty for multidrugresistant patients. But on the basis of our experience, thoracoplasty does not always show good results because sometimes it is difficult to collapse the thick-walled cavities by means of thoracoplasty. Therefore if the patient can tolerate pulmonary resection, you should go for resection instead of thoracoplasty.

Dr Thomas M. Egan (Chapel Hill, NC). Congratulations on outstanding results. How many of your patients had AIDS, and what would be your recommendation for patients with HIV who are infected with tuberculosis?

Dr Shiraishi. We do not have any HIV-positive patients in this series. All patients were HIV-negative. Therefore I do not have a comment on treating HIV-positive patients.

\section{Online-www.aats.org}

Now you can get The Journal of Thoracic and Cardiovascular Surgery online. The Journal online brings you faster delivery time, easy searching of current and back issues, links to PubMed, AATS, WTSA, and other important sites, and more. Visit the Journal online today.

\section{Receive tables of contents by e-mail}

To receive the tables of contents by e-mail, sign up through our Web site at http://www.mosby.com/jtcvs

Choose E-mail Notification

Simply type your e-mail address in the box and click the Subscribe button.

Alternatively, you may send an e-mail message to majordomo@mosby.com.

Leave the subject line blank and type the following as the body of your message: subscribe jtcvs_toc

You will receive an e-mail to confirm that you have been added to the mailing list.

Note that TOC e-mails will be sent out when a new issue is posted to the Web site. 